\title{
Correlation of Balance Ability and Physical Conditions
}

\author{
Yi-Yang Chen, Xu-Yang Gao, and Li-Chi Liao \\ Department of Biomedical Engineering, Ming Chuan University, Taoyuan, Taiwan \\ Email: \{symo960110, lemonsheep66, newstar1015\}@gmail.com \\ Chia-Ling Lee and Chaur-Heh Hsieh \\ Department of Computer and Communication Engineering, Ming Chuan University, Taoyuan, Taiwan \\ Email: jllee@mail.mcu.edu.tw, harrishsieh@gmail.com
}

\begin{abstract}
Generally, students with intellectual disability are not good at athletic, since they have poor balance abilities. The problem causes a bad influence for their daily life and future work. The objective of this paper is to investigate what causes this situation by measuring balance capability with Zebris FDM-System. For comparison, normal college students are also chosen as subjects for the testing. The balance test types include standing with the eyes opened and the eyes closed, and disturbing balance by rotation scheme. The indexes including BMI, leg length and footprint area are adopted as physical conditions. The T-test is used to study the significant differences between normal students and mental disability students (MDs). The results show that there are significant differences in some balance ability index between the students with mental disability and normal students. More importantly, this paper uses correlation and Grey relation to find out the relationship between balance ability and physical conditions. The results by Grey relation show BMI is the most relevant condition influencing equilibrium. However, the correlation measurement reflects that there is significant relevance just for some index.
\end{abstract}

Index Terms-intellectual disability, Zebris FDM-system, balance test, T-test, grey relation, correlation.

\section{INTRODUCTION}

The educational goal of Special Education Vocational school contains three areas of learning, social life and career ability. But we found many students with intellectual disabilities have balance ability problems. The current status of the workplace in employment industry for students with intellectual disabilities is that they can only do some labor works. In order to help those improve the possibility of their future labor job and their athletic abilities, finding the reasons causing their balance problems is the most important thing. Some literatures confirmed that balance ability is affected by some of the subject's physical conditions, status (eye open or eye close) [1], neuromuscular control and performance [2].

Since mental retardation student's health status and balance development significantly are less than normal

Manuscript received December 15, 2016; revised April 27, 2017. students, investigating what causes this situation and promoting their balance ability are necessary. The literature also [3] pointed out that the main factor of poor balance ability of part of mental retardation students is the lack of balance control ability, except the factors of inattention, environment and medical reasons. The study found that body up and down, left and right, back and forth, rotating action to stimulate their vestibular functions to improve balance ability of students with intellectual disability [4]. This paper is devoted to investigating whether equilibrium capability is related to some physical conditions (BMI, leg length and footprint area) [5].

However, equilibrium capability also contains the ability to restore balance state from unbalance one. The lack of this ability can also influence their future life and work especially when emergency happens. Therefore, to explore this problem is also a significant issue. This paper designs an automatic rotating equipment to disturb the balance of users temporarily. It will be used to investigate what causes the balance ability poor.

\section{MATERIALS AND METHODS}

This paper engages in inquiring into the relation between physical conditions and balance ability. Subjects in this paper are from Taoyuan Special Education Vocational school and Ming Chuan University. In this school, we choose some significant mental retardation students as subjects. In Ming Chuan University, we choose some normal fresh men with no balance diseases as subjects.

We use Zebris FDM-System with its specific software (WinFDM-S in windows 7) as the measurement of balance ability. Zebris FDM-System is a device which can measure each part of vertical force on left and right feet. It records the force of each foot of students containing front and backward. Literature in [6] indicated that static balance test standing for 10 seconds with posture stability using force plate had the retest reliability, and the offset trajectories of center of pressure (COP) could be used to effectively detect and quantify postural control parameters, and then to identify static balance performance. The balance test equipment is required to 
connect laptop to display COP of left or right foot [7], [8] In the meantime, students' physical conditions including height, weight, leg length and footprint area are recorded in Excel.

The main procedures of our research are as below:

1. Test static balance and record students' COP.

2. Test balance after rotating and record the recovered time.

3. Analyze the balance ability of all students.

4. Perform T-test to compare balance capability between normal students and mental disability students.

5. Investigate the sequence relevance of physical conditions to equilibrium capability using Grey Relation theory.

6. Find the sequence relevance of physical conditions to balance restored time using Grey Relation theory.

7. Look into the correlation between balance ability and physical conditions.

8. Look into the correlation between recover time and physical conditions

In addition, we design three sections to evaluate their equilibrium capability [9], [10] in the following, as illustrated in Fig. 1.

1. Balance oneself with both legs, eyes open, arms around the chest, keep the posture for ten seconds.

2. Balance oneself with both legs, eyes closed with eye cover put on, arms around the chest, keep the posture for ten seconds.

3. Balance oneself with both legs, eyes open, arms around the chest, keep the posture before turning around three times to disturb one's balance.

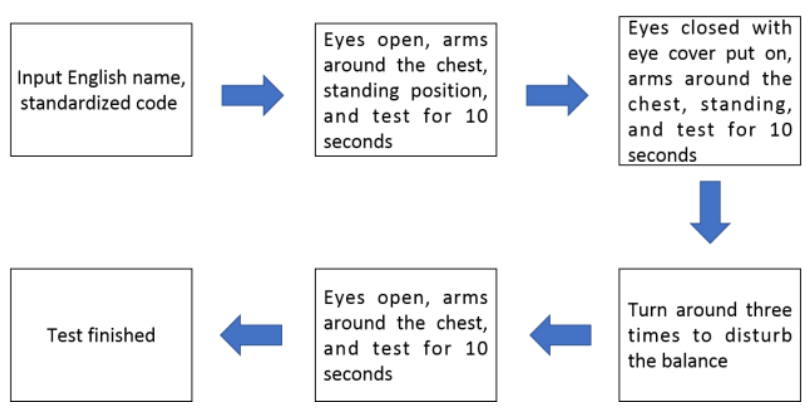

Figure 1. Flow chart of two-leg stance balance test.

\section{ANALYSIS}

This paper aims to study the differences of balance ability between normal students and mental disability students and the correlation between physical conditions. We use T-test, statistic correlation and grey relation as analysis methods. T-test and correlation are finished in SPSS. And the grey relation analysis is performed in the Matlab program written by the authors.

After balance test, we obtain four types of data for both normal students (NS) and mental disability students (MDS) in each section: the force of front left foot, backward left foot, front right foot and backward right foot. This paper measures the following items [11], [12] for NS and MDS, and the results are shown in Table I to Table VI and Fig. 2.

1. The force of left foot minus that of right foot with eyes opened and eyes closed (Table I).

2. Using difference operation to calculate the time to restored balance (Table II).

3. Stability of COP between normal students and MD students (Table III).

4. Correlation between balance ability and BMI, leg length and footprint area (Table IV-V).

5. Correlation between restored balance time and BMI, leg length and footprint area (Table VI).

6. Grey relation results of the sequence of relevance between balance ability and physical conditions.

7. Grey relation results of the sequence of relevance between balance restored time and physical conditions. (Fig. 2)

TABLE I. LEFT ForCe-Right ForCE (MEAN) \& T-Test SCORE

\begin{tabular}{|c|c|c|c|}
\hline & NS & MDS & p-value \\
\hline open eyes & 18.04 & 54.42 & 0.05 \\
\hline close eyes & 23.82 & 56.21 & 0.09 \\
\hline
\end{tabular}

TABLE II. BALANCE RESTORED TIME \& T-TEST

\begin{tabular}{|c|c|c|c|}
\hline & NS & MDS & p-value \\
\hline Time/s & 1.73 & 1.75 & 0.95 \\
\hline
\end{tabular}

TABLE III. STANDARD DEVIATION OF ForCE \& T-TeSt SCORE

\begin{tabular}{|c|c|c|c|}
\hline & NS & MDS & p-value \\
\hline open eyes & 5.54 & 5.75 & 0.8 \\
\hline close eyes & 3.77 & 5.6 & 0.27 \\
\hline
\end{tabular}

TABLE IV. CORRELATION BETWEEN SD/MEAN AND BMI, LEG LENGTH AND FOOTPRINT AREA

\begin{tabular}{|c|c|c|}
\hline & Coefficient & P-value \\
\hline BMI & 0.01 & 0.99 \\
\hline LEG & -0.13 & 0.59 \\
\hline Footprint area & 0.01 & 0.96 \\
\hline
\end{tabular}

TABLE V. CORRELATION BETWEEN LEFT FORCE-RIGHT ForCE (MEAN) AND BMI, LEG LENGTH AND FOOTPRINT AREA

\begin{tabular}{|c|c|c|}
\hline & Coefficient & P-value \\
\hline BMI & 0.59 & 0.01 \\
\hline LEG & -0.05 & 0.84 \\
\hline Footprint area & 0.31 & 0.18 \\
\hline
\end{tabular}

TABLE VI. CORRELATION BETWEEN BALANCE RESTORED TIME AND BMI, LEG LENGTH AND FOOTPRINT AREA

\begin{tabular}{|c|c|c|}
\hline & Coefficient & P-value \\
\hline BMI & -0.14 & 0.56 \\
\hline LEG & 0.04 & 0.87 \\
\hline Footprint area & 0.29 & 0.21 \\
\hline
\end{tabular}




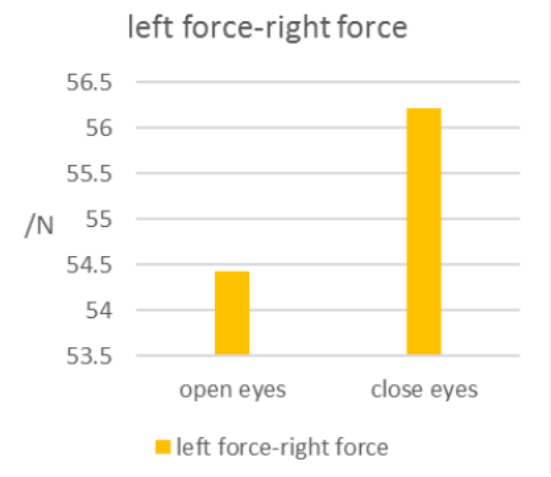

Figure 2. Left force minus right force between open eyes and close eyes for mental disability students.

In order to determine the balance ability, we use absolute value of the difference of restored time between left foot and right foot, and the standard deviation of COP divided by its mean value in each foot, which avoids students just to use one position of foot to keep balance [13]. Normal students' data are used by making t-test to MD students about restored time and stability so as to know if they have significant differences in balance.

At the same time, we want to know whether these data are correlated with BMI and leg length and footprint area. Grey relation analysis is a good method to understand the order of relevance for these conditions [14]-[17]. In addition, correlation is also a good measure to study the relevance between physical conditions and balance ability.

\section{RESULTS AND DISCUSSION}

In this paper, using the t-test between normal students and MD students, we obtain the results that Normal students' values (standard deviation is divided by the mean) are much smaller than those of mental disability students (Fig. 3). It means that the balance ability of normal students is more stable than that of MD students'. And for the index of the force of left foot minus that of right foot, normal students perform much better than MD students (Fig. 4). It shows MD students have serious balance offset especially with eyes closed (Table I). But their balance restored times almost have no significant difference (Table II, Fig. 5). In addition, the capability of balance also has no significant differences with eyes opened. However, we can see in terms of standard deviation of force and T-test with eyes closed, the normal students is better than MD students (Table III).

Furthermore, we found that the equilibrium capability of mental disability students with eyes closed is worse than that with eyes opened (Fig. 2, Fig. 6).

The correlation results reflect that the equilibrium capability just has correlation with BMI, and there is no correlation for these conditions to the balance restored time (Tables IV-VI).

The Grey relation analysis results show that BMI is the most relevant index influencing balance ability, the second is leg length and footprint area (Fig. 7).

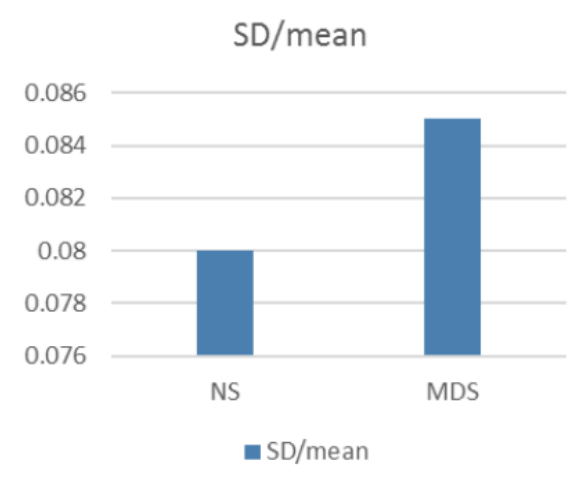

Figure 3. SD/mean between normal students and mental disability students.

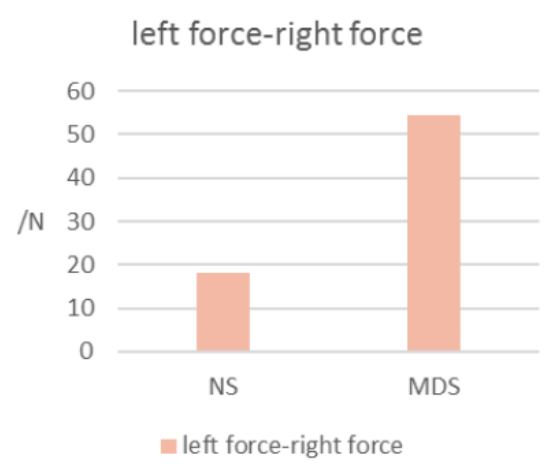

Figure 4. Left force-right force between normal students and mental disability students.

Balance restore time

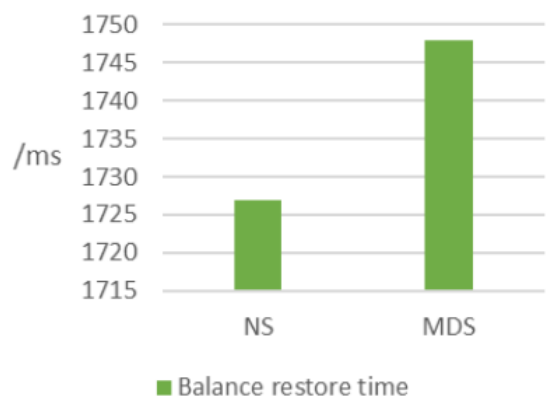

Figure 5. Balance restored time between normal students and mental disability students.

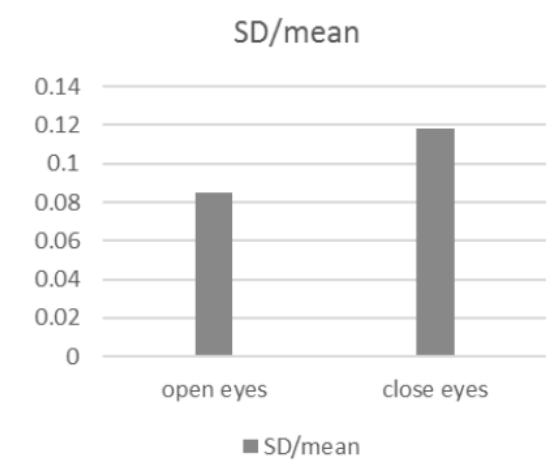

Figure 6. SD/mean between open eyes and close eyes for mental disability students. 


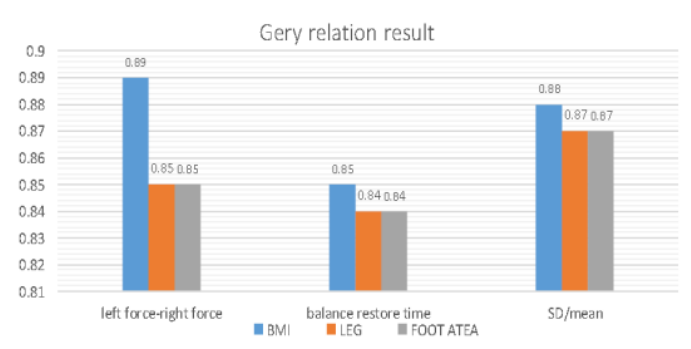

Figure 7. Gery relation result: left force-right force (mean) X BMI, leg length and footprint area.

\section{CONCLUSIONS}

This paper has investigated the correlation of balance ability and physical conditions. We obtained some possible factors (like BMI, leg length) influencing balance ability and balance restored time. We hope our contribution is helpful for other researches. The connection between the balance tests and the body disease which influence balance ability like cerebellar injury is worth further studying in the future.

\section{ACKNOWLEDGMENT}

We would like to express thanks to Hong-Wei Shih for his help in this research, and Ministry of Science and Technology for financial support under Grant MOST 104-2221-E-130-014-MY2.

\section{REFERENCES}

[1] B. L. Riemann and K. Piersol, "Intersession reliability of selfselected and narrow stance balance testing in older adults," Aging. Clin. Exp. Res., pp. 1-4, Nov. 2016.

[2] A. Zech, M. Hübscher, L. Vogt, W. Banzer, F. Hänsel, and K. Pfeifer, "Balance training for neuromuscular control and performance enhancement: A systematic review," J. Athl. Train., vol. 45 , no. 4 , pp. 392-403, 2010.

[3] G. Collum and T. K. Leen, "Form and exploration of medical stability limits in erect stance," Journal of Rehabilitation Research vol. 21, pp. 225-244, 1985.

[4] H. W. Shih, J. J. Xie, C. H. Hsieh, and C. L. Lee, "Design of technical balance training systems," in Proc. Global Conference on Biomedical Engineering, Taipei, June 2016.

[5] T. Baierle, T. Kromer, C. Petermann, P. Magosch, and H. Luomajoki, "Balance ability and postural stability among patients with painful shoulder disorders and healthy controls," $B M C$ Musculoskeletal Disorders, vol. 14, no. 1, pp. 282, 2013.

[6] Y. Yang, F. Pu, Y. Li, S. Li, Y. Fan, and D. Li, "Reliability and validity of kinect RGB-D sensor for assessing standing balance," IEEE Sensors Journal, vol. 14, no. 5, pp. 1633-1638, 2014.

[7] J. H. Choi, B. R. Kim, E. Y. Han, and S. M. Kim, "The effect of dual-task training on balance and cognition in patients with subacute post-stroke," Ann. Rehabil. Med., vol. 39, no. 1, pp. 8190, 2015.

[8] J. R. Yang and S. W. Yang, "An innovation of dynamic balance evaluation system with multi-directions of perturbations," 2001.

[9] C. J. Winstein, E. R. Gardner, D. R. McNeal, P. S. Barto, and D. E. Nicholson, "Standing balance training: Effect on balance and locomotion in hemiparetic adults," Arch. Phys. Med. Rehabil., vol. 70, no. 10, pp. 755-762, Oct. 1989.

[10] Y. J. Gschwind, R. W. Kressig, A. Lacroix, T. Muehlbauer, B. Pfenninger, and U. Granacher, "A best practice fall prevention exercise program to improve balance, strength/power, and psychosocial health in older adults: Study protocol for a randomized controlled trial," BMC Geriatr., vol. 13, no. 1, p. 105, Oct. 2013.
[11] R. J. Butler, G. Bullock, T. Arnold, P. Plisky, and R. Queen, "Research of correlation between footprint parameter analysis and static balance of developmental delay children," Journal of Athletic Training, vol. 51, no. 12, Dec. 2016.

[12] C. L. Chen, "Research of correlation between footprint parameter analysis and static balance of developmental delay children," 2010

[13] S. H. Lee, "The effects of ten-week lower body strength training on balance improvement in female college students," 2014.

[14] J. Tian, B. Yu, D. Yu, and S. Ma, "Clustering-based multiple imputation via gray relational analysis for missing data and its application to aerospace field," The Scientific World Journal, vol. 2013, no. 8, March 2013.

[15] K. Wang, S. Ren, S. Qian, and P. Liu, "Grey relational analysis of benefit of surgical management for abdominal aortic aneurysm," Int. Surg., vol. 99, no. 2, pp. 189-194, 2014.

[16] J. Dai, X. Liu, and F. Hu, "Research and application for grey relational analysis in multigranularity based on normality grey number," The Scientific World Journal, vol. 2014, 2014.

[17] T. Nguyen, A. Bhatti, S. Yang, and S. Nahavandi, "RNA-Seq count data modelling by grey relational analysis and nonparametric gaussian process," PLOS ONE, vol. 11, no. 10, October 26, 2016.

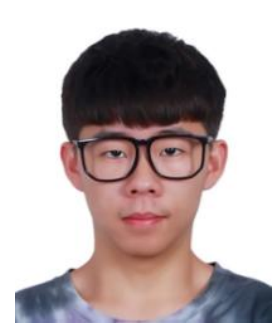

Yi-Yang A. Chen was born in Hangzhou, China, on 10 Jan. Yi-Yang is a student from department of Biomedical Engineering, Ming Chuan University, Taoyuan, Taiwan. He majors in Biomedical signals and Medical imaging. He will graduate in 2018 .

$\mathrm{He}$ is junior now, and he is also an intern research assistant in Center for Cognition and Mind Sciences, National Tsing Hua University and research and teach assistant in Ming Chuan University. In the meantime, he has some papers which is preparing to submit for publication.

Mr. Yi-Yang is a member of Lab for Language and Cognitive Neurology in National Tsing Hua University and Lab Lee in Ming Chuan university. Dr. Chia-Ling Lee(MCU) and Dr. Fan-Pei Gloria Yang(NTHU) are both his advisor.

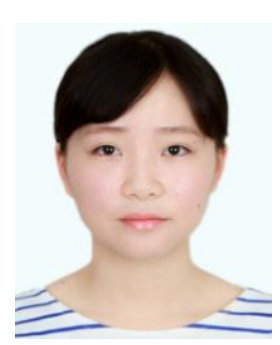

Xu-Yang B. Gao was born in Fuzhou, China, on Sep 10. Xu-Yang and she is a student from Dept. Biomedical Engineering, Ming Chuan university, Taoyuan, Taiwan. She will get a degree in 2018. And her major field study in the biological medicine and biomedical electronics. She is not only a student, but also as a research assistant in MCU.

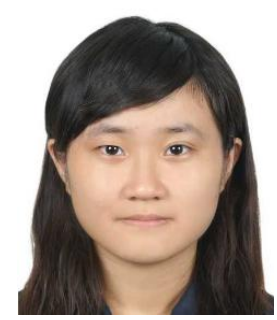

Li-Chi C. Liao was born in Kaohsiung, Taiwan on Jan 28. She is a junior in the department of Biomedical Medical Engineering, Ming Chuan University, Taoyuan, Taiwan at president. She is a member of Lab Lee and also a assistant of professor Chia-Ling Lee.

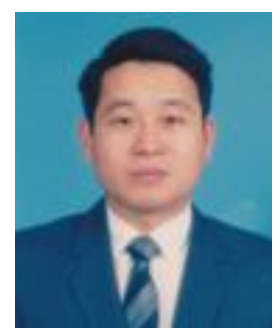

Chia-Ling D. Lee is an Associate Professor in the Department of Biomedical Engineering for the Ming Chuan University in Taoyuan, Taiwan. Dr. Lee received his B.E. in Electrical Engineering from the Taipei Institute of Technology in Taipei and his M.E. in Information and Electronics Engineering and Ph.D. in Electrical Engineering from National Central University in Taoyuan. There, he studied variable structure control design for uncertain interconnected systems. 


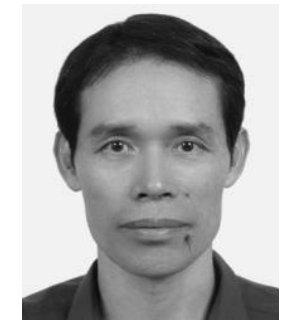

Chaur-Heh E. Hsieh received Ph.D. degree in Electrical Engineering in 1990 from Chung Cheng Institute of Technology (CCIT), Taiwan, Republic of China. In 1981, He joined the faculty of the Department of Electrical Engineering at CCIT, and became a professor in 1993. From 1996 he joined IShou University (ISU) as a full professor of Information Engineering Department. From 1999 to 2002, he served as the chairman of the department. He was a Visiting Scholar in the Department of Electrical Engineering at University of Washington from February to July in 2006. From 2007 he joined Ming-Chuan University as a full professor of Computer and Communication Engineering Department. His research interests include content-based image/video retrieval, sport video analysis, advanced video coding, image watermarking, and deep learning for computer vision. In 2002, he received Outstanding Electrical Engineer Award, Kaohsiung Chapter of The Chinese Institute of Electrical Engineering Society. He is an IET Fellow and IEEE senior member. 